\title{
ON PRIME-LIKE RADICALS
}

\section{S. TUMURBAT and H. FRANCE-JACKSON ${ }^{凶}$}

(Received 17 October 2009)

\begin{abstract}
A radical $\gamma$ is prime-like if, for every prime ring $A$, the polynomial ring $A[x]$ is $\gamma$-semisimple. In this paper, we study properties of prime-like radicals. In particular, we give necessary and sufficient conditions for a radical $\gamma$ containing the prime radical $\beta$ to be prime-like. This allows us to easily find distinct special radicals that coincide on simple rings and on polynomial rings, which answers a question put by Ferrero. It also allows us to reformulate a long-standing open problem of Gardner in terms of prime-like radicals.
\end{abstract}

2000 Mathematics subject classification: primary 16 N80.

Keywords and phrases: prime-like radical, special radical, Amitsur property of radicals, polynomially extensible radicals, subidempotent radicals.

\section{Introduction}

In this paper, all rings are associative and all classes of rings are closed under isomorphisms and contain the one-element ring 0 . The fundamental definitions and properties of radicals can be found in [1,8]. A class $\mu$ of rings is said to be hereditary if $\mu$ is closed under ideals. If $\mu$ is a hereditary class of rings, $\mathcal{U}(\mu)$ denotes the upper radical generated by $\mu$, that is, the class of all rings which have no nonzero homomorphic images in $\mu$. For any class $\mu$ of rings, an ideal $I$ of a ring $A$ is called a $\mu$-ideal if the factor ring $A / I$ is in $\mu$. As usual, for a radical $\gamma$, the $\gamma$ radical of a ring $A$ is denoted by $\gamma(A)$ and the class of all $\gamma$-semisimple rings is denoted by $\mathcal{S}(\gamma) . \pi$ denotes the class of all prime rings and $\beta=\mathcal{U}(\pi)$ denotes the prime radical. The notation $I \triangleleft A$ means that $I$ is a two-sided ideal of a ring $A$. An ideal $I$ of a ring $A$ is said to be essential in $A$ if $I \cap J \neq 0$ for every nonzero two-sided ideal $J$ of $A$. A ring $A$ is called an essential extension of a ring $I$ if $I$ is an essential ideal of $A$. A class $\mu$ of rings is said to be essentially closed if $\mu=\mu_{k}$, where $\mu_{k}=\{A \mid$ $A$ is an essential extension of some $I \in \mu\}$ is the essential cover of $\mu$. A hereditary and essentially closed class of prime rings is called a special class and the upper radical generated by a special class is called a special radical. A hereditary radical containing the prime radical $\beta$ is called a supernilpotent radical. Given a ring $A$, the polynomial

(C) 2010 Australian Mathematical Publishing Association Inc. 0004-9727/2010 \$16.00 
ring over $A$ in a commuting indeterminate $x$ is denoted by $A[x]$. We say that a radical $\gamma$ has the Amitsur property if $\gamma(A[x])=(\gamma(A[x]) \cap A)[x]$ for every ring $A$. A radical $\gamma$ is said to be polynomially extensible if $A[x] \in \gamma$ for every ring $A \in \gamma$. A semiprime ring $R$ is called a $*$-ring [4] if $R / I \in \beta$ for every nonzero ideal $I$ of $R$. The nonnil Jacobson radical ring $W=\{2 x /(2 y+1) \mid x, y \in \mathbb{Z}$ and $(2 x, 2 y+1)=1\}$ is an example of a commutative $*$-ring without minimal ideals, as observed in $[2,3]$. The class of all $*$-rings is denoted by $*$. The importance of the class $*_{k}$ is underlined by the two facts that follow.

THEOREM 1 [3,9]. If $R$ is a nonzero $*$-ring, then the smallest special (respectively, supernilpotent) radical $\widehat{l}_{R}$ (respectively, $\bar{l}_{R}$ ) containing $R$ is an atom in the lattice of all special (respectively, supernilpotent) radicals.

THEOREM 2 [4, Proposition 2]. If $R \in *_{k}$ and $\mu$ is a special class of rings, then $R \in \mathcal{S}(\mathcal{U}(\mu))$ if and only if $R \in \mu$. Thus, in particular, a ring $R \in *_{k}$ is Jacobson semisimple if and only if $R$ is primitive.

A radical $\gamma$ is said to be prime-like if $A[x] \in \mathcal{S} \gamma$ for any prime ring $A$. In this paper, we study properties of prime-like radicals and show their connections with some open problems in radical theory. In particular, we give necessary and sufficient conditions for a radical $\gamma$ containing $\beta$ to be prime-like. This allows us to easily construct pairs of distinct special radicals that coincide on simple rings and on polynomial rings, which answers a question posed by M. Ferrero (see [13]). We also show that the longstanding open question of Gardner [7, Problem 1], which asks whether $\beta=\mathcal{U}\left(*_{k}\right)$, is equivalent to the question of whether the radical $\mathcal{U}\left(*_{k}\right)$ is prime-like. This gives a reason for studying prime-like radicals.

\section{Main results}

We start with some examples of prime-like radicals.

THEOREM 3. The prime radical $\beta$ as well as the smallest special radical $\widehat{l_{R}}$ generated by any commutative $*$-ring $R \in \mathcal{J}$, where $\mathcal{J}$ denotes the Jacobson radical, are primelike.

Proof. If $A \in \pi$, then $A[x] \in \pi \subseteq \mathcal{S} \beta$. Therefore, $\beta$ is prime-like.

Now, let $R \in \mathcal{J}$ be a commutative $*$-ring. Since $\mathcal{J}$ is a special radical, it follows that $\widehat{l_{R}} \subseteq \mathcal{J}$. However, as $R$ is a $*$-ring, it follows from [9] that $\widehat{l_{R}}=\mathcal{U}\left(\pi \backslash \pi_{R}\right)$, where $\pi_{R}$ is the class of all rings $S$ which contain an essential ideal $I$ isomorphic to some accessible subring of $R$. Suppose that $\widehat{l_{R}}(A[x]) \neq 0$ for some prime ring $A$. Then, as $\widehat{l_{R}} \subseteq \mathcal{J}$ and since $\mathcal{J}$ has the Amitsur property [8, Theorem 4.9.26], it follows that $\widehat{l_{R}}(A[x]) \subseteq \mathcal{J}(A[x])=(\mathcal{J}(A[x]) \cap A)[x] \in \mathcal{J}$. Thus, it follows from [8, Proposition 4.9.27] that $0 \neq(\mathcal{J}(A[x]) \cap A)$ is a nil ring. On the other hand, $\mathcal{J}(A[x]) \cap A \in \pi$ since $\mathcal{J}(A[x]) \cap A \triangleleft A \in \pi$ and $\pi$ is hereditary. Moreover, as $A[x] \in \pi$, it follows that $A[x] \in \pi_{R}$ since otherwise $A[x] \in \pi \backslash \pi_{R} \subseteq \mathcal{S}\left(\widehat{l_{R}}\right)$ and we have a contradiction. Then $A[x]$ contains a nonzero ideal $I$ which is isomorphic 
to some accessible subring of $R$. Since $R$ is commutative, so is $I$. However, if a prime ring contains a commutative nonzero accessible subring, then it follows from [15, Lemma 3.2] that the ring is also commutative so $A[x]$ is commutative. But in a commutative ring the set of all nilpotent elements forms the prime radical of the ring, so, as $\mathcal{J}(A[x]) \cap A$ is a nil ring, we have $0 \neq(\mathcal{J}(A[x]) \cap A) \subseteq \beta(A[x])$. But as $A[x] \in \pi$, we have that $\beta(A[x])=0$, which is a contradiction. So $\widehat{l_{R}}(A[x])=0$, which shows that the radical $\widehat{l_{R}}$ is prime-like.

COROLlaRY 4. A polynomially extensible radical $\gamma$ containing $\beta$ is prime-like if and only if $\gamma=\beta$.

PROOF. Let $\gamma$ be a polynomially extensible radical with $\beta \subseteq \gamma$.

If $\gamma=\beta$, then it follows from Theorem 3 that $\gamma$ is prime-like.

Conversely, let $\gamma$ be prime-like and suppose that $\beta \varsubsetneqq \gamma$. Then there exists $0 \neq R \in$ $\gamma \cap \pi$. But then $0 \neq R[x] \in \gamma \cap \mathcal{S} \gamma$ because $\gamma$ is polynomially extensible and primelike, so we have a contradiction. Thus $\gamma=\beta$.

A long-standing open question [7, Problem 1] asks whether $\beta=\mathcal{U}\left(*_{k}\right)$. Since the special radical $\mathcal{U}\left(*_{k}\right)$ is polynomially extensible [5], it follows from Corollary 4 that this problem is equivalent to the following.

PROBLEM 5. Is the upper radical $\mathcal{U}\left(*_{k}\right)$ prime-like?

Our results that follow describe some properties of prime-like radicals.

LEMMA 6. Let $\gamma$ be a prime-like radical. Then $\gamma(A[x])=0$ for every semiprime $\operatorname{ring} A$.

Proof. Let $A$ be a semiprime ring. Then $A$ is a subdirect sum of rings $A / I_{\lambda} \in \pi$, where $\lambda \in \Lambda$ and $\bigcap_{\lambda \in \Lambda} I_{\lambda}=\{0\}$. We will show that $A[x]$ is a subdirect sum of the rings $A[x] / I_{\lambda}[x]$. Suppose that $f(x) \in \bigcap_{\lambda \in \Lambda} I_{\lambda}[x]$ and $f(x)=a_{0}+a_{1} x+\cdots+$ $a_{n} x^{n}$. Then, since $f(x) \in I_{\lambda}[x]$ for every $\lambda \in \Lambda$, it follows that $a_{0}, a_{1}, \ldots, a_{n} \in$ $\bigcap_{\lambda \in \Lambda} I_{\lambda}=\{0\}$. So $f(x)=0$, which shows that $\bigcap_{\lambda \in \Lambda} I_{\lambda}[x]=\{0\}$. Thus $A[x]$ is a subdirect sum of the rings $A[x] / I_{\lambda}[x] \simeq\left(A / I_{\lambda}\right)[x]$. Now, since $A / I_{\lambda} \in \pi$, for every $\lambda \in \Lambda$ and since $\gamma$ is prime-like, it follows that $\left(A / I_{\lambda}\right)[x] \in \mathcal{S}(\gamma)$, for every $\lambda \in \Lambda$. This implies that $A[x] \in \mathcal{S}(\gamma)$ because semisimple classes are closed under subdirect sums.

For a radical $\gamma$, let $\gamma_{x}=\{A \mid A[x] \in \gamma\}$.

A hereditary radical $\gamma$ is said to be subidempotent if the radical class $\gamma$ consists of idempotent rings.

REMARK 7. It follows from [10, Proposition 4.1] that every subidempotent radical $\gamma$ has the Amitsur property and $\gamma_{x}=\{0\}=\beta \cap \gamma$.

We have the following lemma. 
LEMMA 8. If $\gamma$ is a radical with the Amitsur property and if either $\beta \cap \gamma=\gamma_{x}$ or $\gamma_{x}=\{0\}$, then $\gamma$ is prime-like.

PROOF. Let $\gamma$ be a radical with the Amitsur property.

First, consider the case when $\beta \cap \gamma=\gamma_{x}$. Let $A \in \pi$. Then $\gamma_{x}(A) \in \pi \cap \gamma_{x}=$ $\pi \cap \beta \cap \gamma=\{0\}$ so $A \in \mathcal{S} \gamma_{x}$. But, since $\gamma$ has the Amitsur property, it then follows from [14, Theorem 3.5] that $A[x] \in \mathcal{S} \gamma$. Thus $\gamma$ is prime-like.

Now, let $\gamma_{x}=\{0\}$. Then all rings are in $\mathcal{S} \gamma_{x}$ and so, in particular, for any $A \in \pi$, we have $A \in \mathcal{S} \gamma_{x}$. Then, arguing as in the first part of the proof, we get $A[x] \in \mathcal{S} \gamma$, which means that $\gamma$ is prime-like and this ends the proof.

The following is an immediate consequence of Lemma 8 and Remark 7.

COROLlary 9. Every subidempotent radical is prime-like.

We also have the following corollary.

COROLLARY 10. The lower radical $l(\sigma)$ generated by a class $\sigma$ of simple prime rings is prime-like.

PROOF. Since $\sigma$ is hereditary, so the radical $l(\sigma)$ is hereditary too. Moreover, $l(\sigma)$ consists of idempotent rings because all rings in $\sigma$ are idempotent. Thus $l(\sigma)$ is a subidempotent radical and it follows from Corollary 9 that $l(\sigma)$ is prime-like.

We say that a radical $\gamma$ satisfies condition ( $z$ ) if, for every ring $A, A \in \gamma$ implies that $A^{0} \in \gamma$, where $A^{0}$ denotes the ring with the zero multiplication on the additive group of $A$.

Clearly, every radical containing the class of all nilpotent rings, in particular every supernilpotent and every special radical, satisfies condition (z) but subidempotent radicals do not satisfy this condition.

For our next result we need the following fact, which is well known. For completeness, we include a proof, which is simpler than the one given in [12].

Proposition $11[6,12]$. For every radical $\gamma$ and every ring $A$, if $A \in \beta$ and $A^{0} \in \gamma$, then $A \in \gamma$.

Proof. Let $A$ be a ring and let $\gamma$ be a radical such that $A \in \beta$ and $A^{0} \in \gamma$. Since $A \in \beta$ and $A^{0} \in \gamma$ imply $(A / \gamma(A)) \in \beta$ and $(A / \gamma(A))^{0} \simeq\left(A^{0} /(\gamma(A))^{0}\right) \in \gamma$, it suffices to show that if $A^{\circ} \in \gamma$ and $\gamma(A)=0$, then $A=0$. Suppose that $A^{2} \neq 0$ and consider the ideal $T=\{t \in A \mid A t=0\}$ of $A$. Since $A^{2} \neq 0$, it follows that $T \neq A$. Then, as $A \in \beta$, it follows that $0 \neq(A / T) \in \beta$ and so there exists an ideal $I$ of $A$ such that $T \varsubsetneqq I$ and $I^{2} \subseteq T$. Then there exists $t \in I$ such that $A t \neq 0$. Then $(A t)^{2} \subseteq A I^{2} \subseteq A T=0$. Therefore, the mapping $f: A^{0} \rightarrow A t$ given by $f(x)=x t$ is a ring epimorphism, which implies that $A t \in \gamma$ because $A^{0} \in \gamma$. But, as $(A t)^{2}=0$, it follows that $A t \triangleleft$ $A t+A t A$. Therefore, since $A t \in \gamma$, we have that $A t \subseteq \gamma(A t+A t A) \in \gamma$. Now, since $A t+A t A \triangleleft A$, it follows that $\gamma(A t+A t A) \triangleleft A$ and so $\gamma(A t+A t A) \subseteq \gamma(A)$. This implies that $0 \neq A t \subseteq \gamma(A)=0$, which is a contradiction. Thus $A^{2}=0$ and then $A \simeq A^{0} \in \gamma$, which ends the proof. 
PROPOSITION 12. If $\gamma$ is a prime-like radical that satisfies condition ( $z)$, then $\beta \cap \gamma=\gamma_{x}$.

PROOF. Let $\gamma$ be a prime-like radical that satisfies condition (z).

Let $A \in \beta \cap \gamma$. Then $A \in \gamma$ and, by condition (z), we have $A^{0} \in \gamma$. However, since $(A[x])^{0}$ is isomorphic to the direct sum of copies of $A^{0}$, this implies that $(A[x])^{0} \in \gamma$. Moreover, since $A \in \beta$ and since $\beta$ is polynomially extensible [8, p. 275], it follows that $A[x] \in \beta$. Then Proposition 11 implies that $A[x] \in \gamma$, which shows that $A \in \gamma_{x}$. Thus $\beta \cap \gamma \subseteq \gamma_{x}$.

To show that $\gamma_{x} \subseteq \beta \cap \gamma$, let $0 \neq A \in \gamma_{x}$. Then, since $\gamma_{x} \subseteq \gamma$, we have that $A \in \gamma$. Now, if $A \in \beta$, then we are done. So suppose $A \notin \beta$. Then, since $\beta=\mathcal{U}(\pi)$, it follows that $A$ can be homomorphically mapped onto a nonzero $\operatorname{ring} \bar{A} \in \pi$. But then, since $\gamma$ is prime-like, it follows that $\bar{A}[x] \in \mathcal{S} \gamma$. On the other hand, since $A \in \gamma_{x}$ and since $\gamma_{x}$ (being a radical class [8, Theorem 4.9.15]) is homomorphically closed, it follows that $\bar{A} \in \gamma_{x}$. This means that $\bar{A}[x] \in \gamma$ and we have a contradiction. Thus $\gamma_{x} \subseteq \beta \cap \gamma$, which ends the proof.

Note that Remark 7 shows that, even if a radical $\gamma$ has the Amitsur property, the fact that $\beta \cap \gamma=\gamma_{x}$ does not imply that $\gamma$ satisfies condition (z), as subidempotent radicals do not satisfy condition $(\mathrm{z})$.

COROLlaRY 13. Let $\gamma$ be a radical with $\beta \subseteq \gamma$. Then $\gamma$ is prime-like if and only if $\gamma_{x}=\beta$ and $\gamma$ has the Amitsur property.

PROOF. Let $\gamma$ be a prime-like radical with $\beta \subseteq \gamma$.

Then $\gamma$ satisfies condition (z) and Proposition 12 implies that $\beta=\beta \cap \gamma=\gamma_{x}$. To show that $\gamma$ has the Amitsur property, let $A \in \mathcal{S} \gamma_{x}$. Then, since $\gamma_{x}=\beta$, it follows that $A \in \mathcal{S} \beta$. But then it follows from Lemma 6 that $A[x] \in \mathcal{S} \gamma$. This, in view of [14, Theorem 3.5], shows that $\gamma$ has the Amitsur property.

Conversely, let a radical $\gamma \supseteq \beta$ with the Amitsur property be such that $\gamma_{x}=\beta$. Let $A \in \pi$. Then, as $\beta=\mathcal{U}(\pi)$, it follows that $A \in \mathcal{S} \gamma_{x}$. Then, since $\gamma$ has the Amitsur property, it follows from [14, Theorem 3.5] that $A[x] \in \mathcal{S} \gamma$, which shows that $\gamma$ is prime-like.

We are now ready to give our characterization of prime-like radicals $\gamma$ with $\beta \subseteq \gamma$.

THEOREM 14. Let $\gamma$ be a radical with $\beta \subseteq \gamma$. Then $\gamma$ is prime-like if and only if $\gamma(A[x])=(\beta(A))[x]=\beta(A[x])$, for every ring $A$.

PROOF. Let $A$ be any ring and let $\gamma$ be a prime-like radical with $\beta \subseteq \gamma$. It is well known [8, p. 275] that $(\beta(A))[x]=\beta(A[x])$. So it suffices to show that $\gamma(A[x])=(\beta(A))[x]$. Now, since $\gamma$ is a prime-like radical with $\beta \subseteq \gamma$, it follows from Corollary 13 that $\gamma_{x}=\beta$ and $\gamma$ has the Amitsur property. Then $(\gamma(A[x]) \cap A)[x]=$ $\gamma(A[x]) \in \gamma$, which implies that $\gamma(A[x]) \cap A \in \gamma_{x}$. Consequently, $\gamma(A[x]) \cap A \subseteq$ $\gamma_{x}(A)=\beta(A)$. Then $\gamma(A[x])=(\gamma(A[x]) \cap A)[x] \subseteq \beta(A)[x]$. But, since $\beta(A)=$ $\gamma_{x}(A) \in \gamma_{x}$, it follows that $\beta(A)[x] \in \gamma$. Thus, as $\beta(A)[x] \triangleleft A[x]$, it follows that $\beta(A)[x] \subseteq \gamma(A[x])$. Thus $\gamma(A[x])=(\beta(A))[x]$. 
Conversely, let $\gamma$ be a radical with $\beta \subseteq \gamma$ and $\gamma(A[x])=(\beta(A))[x]$, for every ring $A$. Then, since for every prime $\operatorname{ring} A$ we have $\beta(A)=0$, it follows that $\gamma(A[x])=(\beta(A))[x]=0$, which shows that $\gamma$ is prime-like.

Ferrero [13] asked whether two distinct special radicals may coincide on all simple rings as well as on polynomial rings $A[x]$ for all rings $A$. An affirmative answer was given in both [11] and [15]. We will now show that our Theorem 14 provides an easier way of finding special radicals that satisfy Ferrero's requirements.

COROLLARY 15. For any special and prime-like radical $\gamma \nsupseteq \beta$ whose semisimple class contains all prime simple rings (for example, $\widehat{l}_{W}$ is such a radical), the prime radical $\beta$ and the radical $\gamma$ satisfy Ferrero's requirements.

PROOF. Since $\gamma$ is special and prime-like, it follows from Theorem 14 that $\gamma(A[x])=$ $\beta(A[x])$, for every ring $A$. Let $A$ be a simple ring. Then either $A^{2}=0$ or $A^{2}=A \in \pi$. In the first case, $A \in \beta \varsubsetneqq \gamma$ so $\beta(A)=A=\gamma(A)$. In the second case, $\beta(A)=0=$ $\gamma(A)$ since all simple prime rings are in $\mathcal{S} \gamma$, which ends the proof.

It is well known [1, Theorem 1, p. 233] that the collection $\boldsymbol{L}_{s}$ of all special radicals forms a complete lattice with respect to inclusion of radical classes, where the meet and the joint of a family of special radicals $\gamma_{\lambda}, \lambda \in \Lambda$, are defined by $\bigwedge_{\lambda \in \Lambda} \gamma_{\lambda}=\bigcap_{\lambda \in \Lambda} \gamma_{\lambda}$ and $\nabla \lambda \in \Lambda \gamma_{\lambda}=\mathcal{U}\left(\bigcap_{\lambda \in \Lambda}\left(\mathcal{S}_{\gamma_{\lambda}} \cap \pi\right)\right)$, respectively. We conclude the paper with the following theorem.

THEOREM 16. The collection $\boldsymbol{L}_{s} \boldsymbol{p l}$ of all special and prime-like radicals is a complete sublattice of the lattice $\boldsymbol{L}_{s}$ of all special radicals. The radical $\widehat{l}_{W}$ is an atom of $\boldsymbol{L}_{s p l}$.

PROOF. Let $\gamma_{\lambda}, \lambda \in \Lambda$ be a family of special and prime-like radicals. Then it follows from Corollary 13 that, for every $\lambda \in \Lambda$, we have that $\left(\gamma_{\lambda}\right)_{x}=\beta$ and $\gamma_{\lambda}$ has the Amitsur property. Then, it follows from [15, Proposition 3.9] that $\bigwedge_{\lambda \in \Lambda} \gamma_{\lambda}$ has the Amitsur property. We will show that $\left(\bigwedge_{\lambda \in \Lambda} \gamma_{\lambda}\right)_{x}=\beta$. Since $\bigwedge_{\lambda \in \Lambda} \gamma_{\lambda}$ is a special radical, it follows from [8, Proposition 4.9.20] that so is $\left(\bigwedge_{\lambda \in \Lambda} \gamma_{\lambda}\right)_{x}$. Thus $\beta \subseteq\left(\bigwedge_{\lambda \in \Lambda} \gamma_{\lambda}\right)_{x}$. Now, let $A \in\left(\bigwedge_{\lambda \in \Lambda} \gamma_{\lambda}\right)_{x}$. Then $A[x] \in \bigwedge_{\lambda \in \Lambda} \gamma_{\lambda}$ so $A[x] \in \gamma_{\lambda}$ for every $\lambda \in \Lambda$. This means that $A \in\left(\gamma_{\lambda}\right)_{x}$ for every $\lambda \in \Lambda$ and, since $\left(\gamma_{\lambda}\right)_{x}=\beta$, it follows that $A \in \beta$. Thus $\left(\bigwedge_{\lambda \in \Lambda} \gamma_{\lambda}\right)_{x}=\beta$ and so it follows from Corollary 13 that $\bigwedge_{\lambda \in \Lambda} \gamma_{\lambda}$ is prime-like.

We will now show that $\nabla \lambda \in \Lambda \gamma_{\lambda}$ is prime-like. Let $A \in \pi$. Then $A[x] \in \pi$. Moreover, since each $\gamma_{\lambda}, \lambda \in \Lambda$ is prime-like, it follows that $A[x] \in \mathcal{S} \gamma_{\lambda}$ for every $\lambda \in \Lambda$. Thus $A[x] \in \bigcap_{\lambda \in \Lambda}\left(\mathcal{S} \gamma_{\lambda} \cap \pi\right) \subseteq \mathcal{S}\left(\mathcal{U}\left(\bigcap_{\lambda \in \Lambda}\left(\mathcal{S} \gamma_{\lambda} \cap \pi\right)\right)\right)$. Thus $A[x] \in$ $\mathcal{S}\left(\nabla \lambda \in \Lambda \gamma_{\lambda}\right)$, which shows that $\nabla \lambda \in \Lambda \gamma_{\lambda}$ is prime-like.

It follows from Theorem 1 that $\widehat{l}_{W}$ is an atom of $\boldsymbol{L}_{s}$. Moreover, Theorem 3 implies that $\widehat{l}_{W}$ is a prime-like radical. Thus, since $\boldsymbol{L}_{s \boldsymbol{p}}$ is a complete sublattice of $\boldsymbol{L}_{s}$, it follows from Theorem 16 that $\widehat{l}_{W}$ is an atom of $\boldsymbol{L}_{s \boldsymbol{p}}$, which ends the proof.

\section{References}

[1] V. A. Andrunakievich and Yu. M. Ryabukhin, Radicals of Algebra and Structure Theory (Nauka, Moscow, 1979) (in Russian).

[2] H. France-Jackson, ‘*-rings and their radicals', Quaestiones Math. 8 (1985), 231-239. 
[3] H. France-Jackson, 'On atoms of the lattice of supernilpotent radicals', Quaestiones Math. 10 (1987), 251-255.

[4] H. France-Jackson, 'Rings related to special atoms', Quaestiones Math. 24 (2001), 105-109.

[5] H. France-Jackson, 'On supernilpotent radicals with the Amitsur property', Bull. Aust. Math. Soc. 80 (2009), 423-429.

[6] B. J. Gardner, 'Sub-prime radicals determined by zerorings', Bull. Aust. Math. Soc. 12 (1975), 95-97.

[7] B. J. Gardner, 'Some recent results and open problems concerning special radicals', in: Radical Theory (Proceedings of the 1988 Sendai Conference, Sendai, 24-30 July 1988), (ed. S. Kyuno) (Uchida Rokakuho Pub. Co. Ltd, Tokyo, Japan, 1989), pp. 25-56.

[8] B. J. Gardner and R. Wiegandt, Radical Theory of Rings (Marcel Dekker Inc., New York, 2004).

[9] H. Korolczuk, 'A note on the lattice of special radicals', Bull. Pol. Acad. Sci. Math. 29 (1981), 103-104.

[10] N. V. Loi and R. Wiegandt, 'On the Amitsur property of radicals', Algebra Discrete Math. 3 (2006), 92-100.

[11] S. Tumurbat, 'On special radicals coinciding on simple rings and on polynomial rings', J. Algebra Appl. 2(1) (2003), 51-56.

[12] S. Tumurbat and R. Wiegandt, 'Principally left hereditary and principally left strong radicals', Algebra Colloq. 8(4) (2001), 409-418.

[13] S. Tumurbat and R. Wiegandt, 'A note on special radicals and partitions of simple rings', Comm. Algebra 30(4) (2002), 1769-1777.

[14] S. Tumurbat and R. Wiegandt, 'Radicals of polynomial rings', Soochow J. Math. 29(4) (2003), 425-434.

[15] S. Tumurbat and R. Wiegandt, 'On radicals with Amitsur property', Comm. Algebra 32(3) (2004), 1219-1227.

S. TUMURBAT, Department of Algebra, University of Mongolia, PO Box 75,

Ulaan Baatar 20, Mongolia

e-mail: stumurbat@hotmail.com

H. FRANCE-JACKSON, Department of Mathematics and Applied Mathematics, Nelson Mandela Metropolitan University, Summerstrand Campus (South),

PO Box 77000, Port Elizabeth 6031, South Africa

e-mail: cbf@easterncape.co.uk 